\title{
Evaluación de la fenología reproductiva y dinámica de producción del cacao (Theobroma cacao L.) clon CCN - 51
}

\author{
Evaluation of Reproductive Phenology and Dynamics of Production Cacao \\ (Theobroma cacao L.) clone CCN - 51
}

Cindy Paola Castro Muñoz ${ }^{1}$, Nadia Masaya Panduro Tenazoa², Ena Vilma Velazco Castro², Elsa Scarlet Iturraran Pinto ${ }^{3}$

\section{RESUMEN}

En el Fundo Santa Rosita, ubicado en el Distrito de Irazola, con el objetivo de evaluar la fenología reproductiva y dinámica de la producción del cacao (Theobroma cacao L) clon CCN - 51, se seleccionaron de forma aleatoria, 20 plantas injertas de 10 años de edad, en un área de 1 ha. Se evaluó, el crecimiento de flores $(\mathrm{cm})$, frutos $(\mathrm{cm})$, rendimiento (Kgha-1), raíz $(\mathrm{cm})$, concentración de nutrientes (\%, para macronutrientes y mgKg-1, para micronutrientes), en las fases fenológicas de floración, fructificación y maduración. La medición de las flores y frutos se efectuó con una regla milimetrada y un vernier digital, la evaluación de la raíz se realizó a través de rizotrones, semanalmente; se efectuó un análisis foliar por cada fase fenológica y un análisis de suelo al inicio y al final del experimento. Las flores presentaron una longitud inicial de $0.4 \pm 0.1 \mathrm{y}$ final de $1.5 \pm 0.01 \mathrm{~cm}$, los frutos mostraron una longitud inicial de $1.9 \pm 0.6$ y final de $19.4 \pm 2.4 \mathrm{~cm}$, con un diámetro inicial de $0.5 \pm 0.1$ y final de 8.4 $\pm 0.9 \mathrm{~cm}$; la raíz empezó a emerger desde los 7 días, hasta 105 días después de la instalación, el crecimiento radicular fue de $2.8 \pm 0.73 \mathrm{~cm}$, el rendimiento en la parcela fue de $607.61 \pm 318.7 \mathrm{Kg} / \mathrm{ha}$; encontrándose dentro del rango aceptable, las concentraciones de los nutrientes fue suficiente para calcio, magnesio, manganeso y zinc e insuficiente para nitrógeno, fósforo, potasio, fierro y cobre.

Palabras claves: Fenología, flor, fruto, rendimiento, raíz, rizotron, concentración.

\begin{abstract}
In the Fundo Santa Rosita located in the District of Irazola, with the objective of evaluating the reproductive phenology and dynamics of production of cocoa (Theobroma cacao L) clone CCN - 51, they were selected at random, 20 grafted plants 10 years old, in an area of 1 ha. We evaluated the growth of flowers $(\mathrm{cm})$, fruits $(\mathrm{cm})$, performance (Kgha-1), root $(\mathrm{cm})$, nutrient concentration (\%, for macronutrients and mgkg-1 for micronutrients), in phenological phases flowering, fruiting and ripening. Measuring flowers and fruits are made with a millimeter ruler and a digital vernier, evaluation was performed root through rhizotrons, weekly; Foliar analysis per phenological phase and a soil test at the beginning and end of the experiment was conducted. Flowers had an initial length of $0.4 \pm 0.1$ and $1.5 \pm$ end of $0.01 \mathrm{~cm}$, the fruits showed an initial length of $1.9 \pm 0.6$ and $19.4 \pm 2.4$ final $\mathrm{cm}$, with an initial diameter of $0.5 \pm 0.1$ and $8.4 \pm 0.9$ end $\mathrm{cm}$; Root began to emerge from 7 days to 105 days after installation, the root growth was $2.8 \pm 0.73 \mathrm{~cm}$, the yield on the plot was $607.61 \pm 318.7 \mathrm{Kg} / \mathrm{ha}$; being within the acceptable range, nutrient concentrations was sufficient to calcium, magnesium, manganese and zinc and insufficient to nitrogen, phosphorus, potassium, iron and copper.
\end{abstract}

Keywords: Phenology, flower, fruit, root, yield, Rhizotron, concentration.

\footnotetext{
${ }^{1}$ Universidad Nacional Intercultural de la Amazonia. Email: cpcastrom@hotmail.com

${ }^{2}$ Docente. Universidad Nacional Intercultural de la Amazonia.

${ }^{3}$ Universidad Nacional de Ucayali.
} 


\section{INTRODUCCIÓN}

El Perú, presenta condiciones agro ecológicas ideales para la producción de cacao de alta calidad; la exportación se centra en productos como la manteca de cacao (representa el $50 \%$ ) y en menor escala el chocolate, pasta de cacao, cacao en polvo y granos de cacao (MINAG, 2010).

Hasta el presente, diversas investigaciones se han centrado intensamente en el estudio agronómico o botánico de la parte aérea del árbol de cacao; sin embargo, del conocimiento del sistema radicular en relación a la fenología reproductiva, se carece de información; pese a que ayudará enormemente en el desarrollo de las prácticas agronómicas tales como operaciones de labranza, drenaje, aplicación de fertilizantes entre otras labores; que imprescindiblemente exigen conocer no sólo el desarrollo y las actividades de las raíces sino saber cómo éstas son modificadas por el medio, (Mejía, 1949).

Por lo expuesto es de importancia estudiar la fenología del cultivo de cacao y su relación con el desarrollo radicular, el conocimiento de éstos parámetros, juegan un papel importante en la adquisición de agua y nutrientes, competencia entre plantas, composición, distribución y desarrollo de la biota en el suelo. Características que son de vital importancia para la aplicación de prácticas agrícolas que den respuesta a las reales necesidades; permitiendo que el cultivo tradicional en un breve tiempo incremente su productividad (Hormoza, 2007). En tal sentido, el presente estudio persigue el objetivo de evaluar el comportamiento de la fenología reproductiva y la dinámica de producción en el cultivo de cacao (Theobroma cacao L.) CCN - 51, en una parcela agroforestal, en el Distrito de Irazola.

\section{MATERIALES Y MÉTODOS}

El proyecto se estableció en el fundo Santa Rosita, ubicado a $500 \mathrm{~m}$, de la carretera Tahuantinsuyo a 2.5 $\mathrm{Km}$, de la carretera Federico Basadre, Km 113, en el Distrito de Irazola, Provincia Padre Abad, Región Ucayali-2014.

La plantación se encuentra en un suelo franco arcilloso con un $\mathrm{pH}$ medianamente acido en un rango de 5.7 y con una fertilidad natural media.

Esta plantación se estableció en una parcela demostrativa de un sistema de producción agroforestal, instalado por el Centro Mundial de Agroforestería (ICRAF), tomando como componente principal al cacao (Theobroma cacao L.), además de caoba (Swietenia macrophylla), capirona (Calycophyllum spruceanum), pijuayo (Bactris gasipaes), guaba (Inga edulis) y con cobertura de ushpika (Conmelina spp.).

Se eligieron 20 plantas de cacao, instaladas en campo definitivo a un distanciamiento de $3 \mathrm{~m} \times 3 \mathrm{~m}$, la selección fue aleatoria dentro de una parcela de 1 ha, teniendo en cuenta el mismo estado fenológico (70\% de floración).

En los árboles seleccionados, se escogió y marcó una rama de cada punto cardinal (norte, este, oeste y sur). De cada rama se escogió cinco cojines florales y dentro de estos cojines se eligieron cinco flores a las cuales se midió su longitud, desde que empezaron a emerger hasta su apertura.

Las mediciones y observaciones del hábito de los frutos, se realizaron sobre las mismas ramillas elegidas para el estudio del desarrollo reproductivo. Se midieron la longitud y diámetro, desde la fecundación.

Para el obtener el rendimiento, las mazorcas fueron partidas con un machete, posteriormente se extrajeron los granos y con las manos se frotó la semilla hasta retirar el mucilago o pulpa, posteriormente se secó y pesó.

El rizotron se ubicó, con orientación hacia el este. La instalación fue en la mitad de la proyección de la copa de la planta de cacao, se efectuó una perforación de $40 \mathrm{~cm} \times 40 \mathrm{~cm} \times 40 \mathrm{~cm}$, posteriormente se colocó el rizotrón de manera fija, quedando un espacio de $20 \mathrm{~cm}$ para la evaluación. En esta labor se usó una lámina trasparente y plumones de $0.6 \mathrm{~mm}$, de colores negro, rojo, azul y verde, que sirvió para dibujar las raíces y diferenciarlas de cada observación. Se escanearon los papeles filminas junto a un papel milimetrado de tamaño A4. Se utilizó el programa AUTO CAD versión 2011, con la finalidad de obtener medidas exactas de cada una de las raíces emergidas, posteriormente se midió la longitud de cada raíz encontrada. Por otra parte, la tasa de crecimiento se estableció a partir de la diferencia entre la última medición y la medición anterior, (Calvert, 1993).

Para la concentración de nutrientes, se efectuó el muestro foliar en cada fase fenológica (floración, fructificación, maduración de frutos y brotamiento); se colectó la muestra según las recomendaciones de Carrillo, (2006); y su conservación según, el procedimiento brindado por Sadzawka et al, (2007).

\section{RESULTADOS YDISCUSIÓN}

\section{Crecimiento de botones florales}

La figura 1, muestra el crecimiento de los botones florales, iniciando a los 7 días después de la instalación (ddi) y esta se prolonga hasta los 28 días con una longitud inicial de $0.4 \pm 0.1 \mathrm{~cm}$ y final de $1.5 \pm$ $0.1 \mathrm{~cm}$, con un crecimiento de $0.3 \mathrm{~cm}$ por semana; posteriormente, inicia la apertura de los sépalos para la fecundación y cuajado de los frutos. De las flores, el $79 \%$ se desprendieron del tallo, $15 \%$ lograron la apertura, 7 \% cuajó y el $1 \%$ llegó a ser fruto. Coincidiendo con MINAG (2011), que aduce que el cacao tiene un desarrollo floral de 30 días en promedio, tiempo en que la flor obtiene $1.5 \mathrm{~cm}$ de longitud. Horowitz (1982), hace hincapié en que el proceso de abscisión comienza entre los 13 y 22 días después de iniciada la floración y en general se estima que con frecuencia se desprende el 50 al $75 \%$ de las flores. 


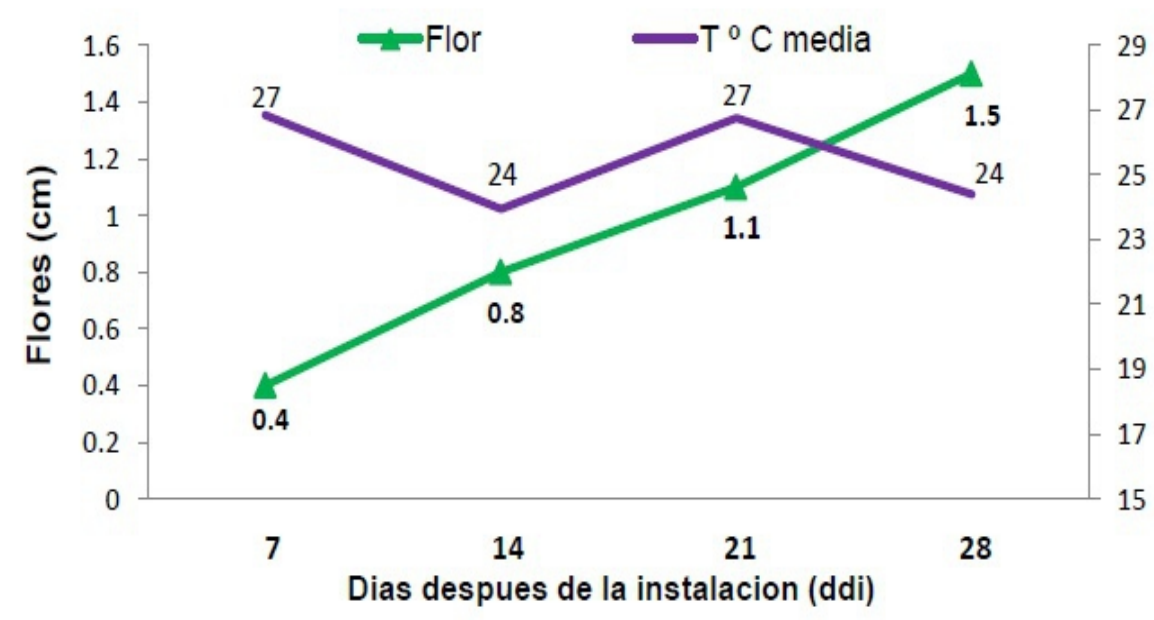

Figura 1. Crecimiento de flores de cacao CCN - 51 y temperatura, Distrito de Irazola, Ucayali, 2014.

\section{Crecimiento de frutos}

En la figura 2, se observa que los frutos inician su desarrollo a los 35 días después de la instalación, hasta los 175 días, longitud inicial de $1.9 \pm 0.6$ y final de $19.4 \pm 2.4 \mathrm{~cm}$, diámetro inicial de $1.0 \pm 0.5 \mathrm{y}$ final de 8.4 \pm 0.9 ; en el transcurso del estudio, los frutos obtuvieron un crecimiento de $0.9 \mathrm{~cm}$ de longitud y 0.4 $\mathrm{cm}$ de diámetro promedio por semana. De los frutos evaluados el $88.1 \%$ no logró desarrollarse a causa de una marchitez prematura y perforaciones causadas por monos y aves, el 11.9 \% fue cosechado. Hardy (1961), reporta que los frutos padecen de marchitamiento prematuro, iniciado con secamiento y arrugamiento, esto ocurre cuando los frutos tienen unos 50 días, o una longitud de $6 \mathrm{~cm}$ de largo, la proporción de frutos afectados varía grandemente de árbol a árbol; con un porcentaje que oscila entre el $19 \mathrm{y}$ el $92 \%$.

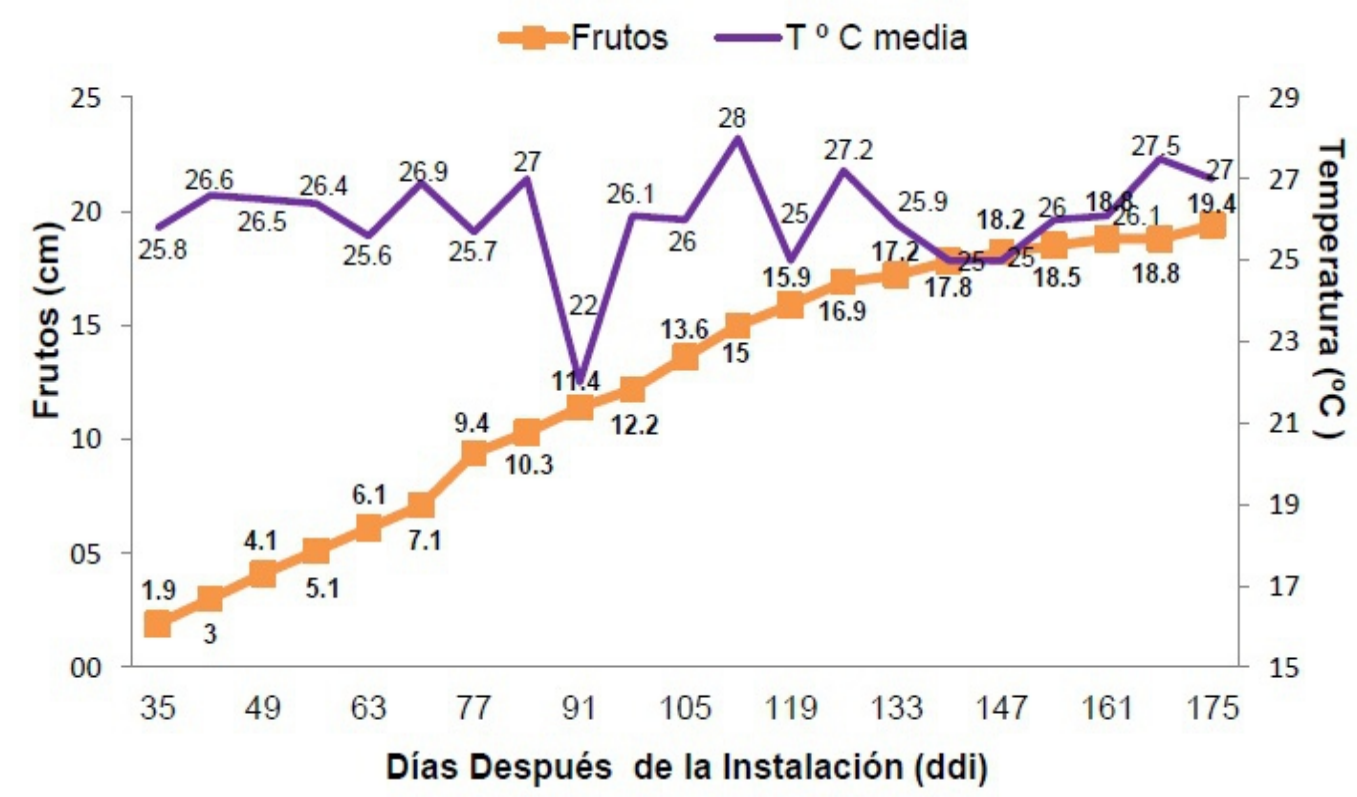

Figura 2. Crecimiento de frutos de cacao CCN - 51 y temperatura, Distrito de Irazola, Ucayali, 2014.

\section{Rendimiento}

A una densidad de siembra por hectárea de $3 \mathrm{~m} \times 3 \mathrm{~m}$ y un total de 1111 plantas, en esta plantación se ha cosechado $9 \pm 4.5$ mazorcas por planta, el cual resulta en $0.546 \pm 286.8 \mathrm{Kg}$ de semilla seca por planta; es decir, $607.61 \pm 318.7 \mathrm{Kg}$ semilla seca por hectárea. Estudios realizados por MINAG y DEVIDA (2012), reportan que el cacao $\mathrm{CCN}-51$, tiene una producción que oscila de 937 a $2812 \mathrm{Kg}$ por hectárea, en una plantación con manejo tradicional; por otro lado
Rosero y Toapanta (2008), menciona que una plantación de cacao de la mismo clon, con manejo orgánico, muestra un rendimiento de $300 \mathrm{Kg}$.ha.

\section{Crecimiento radicular}

En la figura 3, se observa el crecimiento radicular y el desarrollo de las flores tienen una estrecha relación; las flores evaluadas mantuvieron un normal crecimiento y una duración de 28 días, el crecimiento de la raíz empieza a emerger a los $7 \mathrm{ddi}$, hasta 
alcanzar su primer pico a los 28 ddi. Así mismo, se observa que los frutos inician su desarrollo desde los 35 hasta los 119 ddi. Del mismo modo, la raíz mostró un crecimiento de dos picos en esta fase, el primero a los 77 y el segundo a los $91 \mathrm{ddi}$, posteriormente el crecimiento de la raíz se detiene a los 105 ddi, hasta el final del estudio. Las raíces dejaron de crecer, antes que se iniciara la fase de maduración de los frutos. La fase de maduración se dio desde los 119 hasta los 175 ddi, con una duración de 28 días. Coincidiendo con lbacache (1994), quien menciona que la raíz dejó de crecer cuando en la parte aérea de la planta se desarrollaba la fase de fructificación. Ibacache (1999) en su estudio del olivo manifestó que la periodicidad en el crecimiento de raíces depende en gran medida del crecimiento de brotes y del nivel de producción de fruta en la plantas.

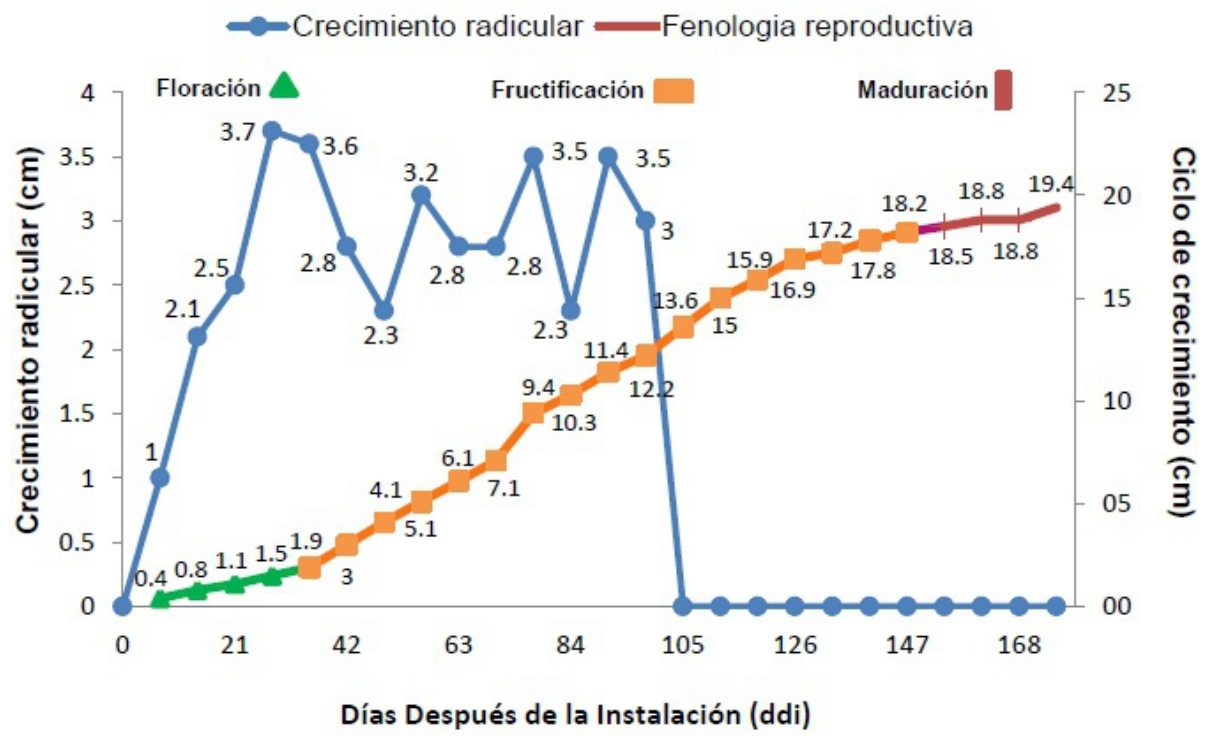

Figura 3. Crecimiento radicular y fenología reproductiva de cacao $\mathrm{CCN}-51$, Distrito de Irazola,

\section{CONCLUSIONES}

El crecimiento de las flores se caracterizó por tener un periodo de $28 \mathrm{ddi}$; con un crecimiento de $1.5 \mathrm{~cm}$ de longitud. Las condiciones climáticas de temperatura y humedad relativa, influyeron en el crecimiento de los frutos desarrollándose desde los 35 a los 175 ddi, en este proceso se apreció la fase de fructificación con 119 días y la fase de maduración con 28 días, con una longitud de $19.4 \mathrm{~cm}$ y un diámetro de $8.4 \mathrm{~cm}$.

El rendimiento del cultivo de cacao (Theobroma cacao) del clon $\mathrm{CCN}$ - 51, en una parcela agroforestal de 10 años de edad y bajo un manejo orgánico fue de $607.61 \mathrm{Kg}$ por hectárea, en un periodo de 175 días.

La raíz tuvo un crecimiento de $2.8 \mathrm{~cm}$ por semana, presentando tres picos de crecimiento a los 28, 77 y 91 ddi, y dejó de crecer desde los 105 hasta los 175 ddi, influenciado por la frecuencia de lluvias, presentes en cada fase evaluada.

Aun cuando la plantación de cacao CCN - 51, ostentó un suelo de textura franco arcilloso, $\mathrm{pH}$ medianamente ácido, fertilidad media y concentración de macronutrientes primarios en rango crítico, estos fueron suficientes para lograr un rendimiento superior a los establecidos bajo un manejo orgánico.

\section{AGRADECIMIENTO}

A la Universidad Nacional Intercultural de la Amazonia y a las distintas instituciones que brindaron un esencial aporte en la ejecución de este trabajo de investigación.

\section{BIBLIOGRAFÍA}

Calvert, E. (1993). Aproximación al Ciclo Fenológico del Palto (Persea americana Mill), Cultivar Fuerte, para la Zona de Quillota. V Región. Taller de titulación. Quillota - Chile. Universidad Católica de Valparaíso Facultad de Agronomía. 143 p.

Carrillo, M. (2006). El Muestreó Foliar en Cacao. Departamento de Manejo de Suelos - INIAP. Quevedo - Los Ríos - Ecuador. 2 p.

Gaspar, L. (2008). Fertilización del cultivo de la vid. Agro estrategias - Consultores. Consultado 01/03/2015. Disponible en: http://www.agroestrategias.com.pdf

Hardy, F. (1961). Manual del cacao. Instituto Interamericano de Ciencias Agrícolas. Editorial Antonio Leheman. Turrialba - Costa rica.

Hormoza, P. (2007). Utilizacion de Rizotrones para el Estudio de la Dinámica del Crecimiento de Raíces de Palma de Aceite. Corporación Centro de Investigación en Palma de Aceite - CENIPALMA. Bogotá-Colombia.

Horowitz, H. (1992). Influence des conditions de milieu sur la formation et la chute des organes floraux chez le cotonnier. 311-342 p. 
Ibacache, A; Tapia, F; Olivares, P. (1999). Estudio de la fenología aérea y radicular del Olivo. Chile. 78 p.

Ibacache, A; Lobato, A; Rojas, N y Jopia, C. (1994). CHIRIMOYO - Novedoso Sistema para Medir el Crecimiento de Raíces. Revista Tierra Adentro. Chile. 4 p.

Mejía, L. (2000). Nutrición del cacao. Publicación de Corpoica. Tecnología para el Mejoramiento del Sistema de Producción de Cacao. Corporación Colombiana de Investigación Agropecuaria, Bucaramanga, Colombia.

Ministerio de Agricultura (MINAG) y Comisión Nacional para el Desarrollo y Vida sin Drogas (DEVIDA). (2012). Catálogo de Cultivares de Cacao en el Perú. $112 \mathrm{p}$.

Ministerio de Agricultura (MINAG). (2011). Condiciones Agroclimáticas del Cultivo del Cacao. Cartilla No 13. Perú. 3 p.
Ministerio de Agricultura (MINAG). (2010). Cacao Peruano Conquistando el Perú "IV Congreso Nacional". 2 p.

Rosero, A y Toapanta, R. (2008). Diseño de Una Maquina Separadora de la Semilla de Cacao. Escuela Politécnica Nacional. Facultad de Ingeniería Mecánica Ecuador-Quito. 8 p.

Sadzawka, A; Carrasco, M; Demanet, R; Flores, H: Grez, R; De la Luz, M y Neaman, A. (2007). Métodos de Análisis de Tejidos Vegetales. Instituto de Investigaciones Agropecuarias. Chile. 120 p.

Sánchez, A. (2011). Manual para la producción de un cacao de calidad. Primera edición. Ucayali - Perú. $13 p$.

Uson, A. Boixadera, J. Martin, A. (2010). Tecnología de suelos: estudio de casos. Editores Zaragoza prensa universitaria de Zaragoza. España. 515 p. 